\title{
Removal of residual stresses in aircraft structural elements by pulsating subsonic gas flows treatment
}

\author{
Gennadiy Kovalenko ${ }^{1}$, and Denis Ivanov ${ }^{1, *}$ \\ ${ }^{1}$ Saint Petersburg state University of civil aviation, Saint Petersburg, Russia
}

\begin{abstract}
The paper presents a promising method of removing undesirable tensile residual stresses in aircraft metal structural elements; the basis of the method is the action of pulsating subsonic gas flows on the surface of these elements. The results of the research showed high effectiveness of controlling residual stresses in aircraft metal structural elements by using pulsating subsonic air flows.
\end{abstract}

Traditionally, the residual stresses, in particular dangerous tensile ones in the surface layers, which appear during manufacturing or corrective maintenance, are reduced by heating. This method has a range of considerable shortcomings. Along with the problem of granules coagulation, and if there are carbide or intermetallic inclusions, it is necessary to mention that the residual stresses are removed to the minimum value at the given temperature during 1.5-2 hours, thus considerably rising the cost of manufacturing or parts' maintenance as well as their duration. The methods of surface plastic deformation are not suitable for residual stresses removal in the parts of aircraft engines, because bead blasting and centrifugal-ball (rolling cold hardening) inadmissibly worsen the surface smoothness; while rolling by a steel ball (roller) and a diamond smoothing by arbor are hardly implemented for the parts of a complicated shape, as well as lead to the change of linear dimensions.

The application of non-deformative methods for controlling residual stresses in aircraft structural elements seems to be having prospects. These methods include the action of pulsating subsonic air flows (gas-impulsive treatment) in whole, and do not have the mentioned above shortcomings.

The stresses, which exist and balance in the solid after determining the causes of their appearance, are accepted to be called residual stresses. Residual stresses are always internal because they balance themselves inside the solid or hard aggregate. The generation of residual stresses is always connected with heterogeneous plastic deformations in adjacent dimensions of the material or the aggregate, caused by the external deformative action, by temperature gradient along the section of the product during quick cooling, or by the heterogeneity and irregularity of phase transformations, connected with the volume change.

The goal of mechanical methods of determining residual stresses is to define on the basis of the known set of movements or deformation values what has caused their stresses.

* Corresponding author: ivanov.denis.71@mail.ru 
The research of residual stresses was carried by Davidenkov's method, applied to the ring samples.

In the ring samples the values of tangential residual stresses decrease from the surface to the centre, and after going through the neutral layer, they change their sign into the opposite one. During the distribution of residual stresses on the thickness of the ring, which is close to the linear one, in the layer, remote from the surface for distance a

$$
\sigma_{\theta(\mathrm{a})}= \pm 2 \frac{E \delta_{c}}{\left(1-\mu^{2}\right) D_{a v}^{2}}(h / 2-a),
$$

where E isYoung's modulus;

$\mu$ is Poisson's ratio;

$\mathrm{D}_{\mathrm{av}}$ is an average diameter of the ring before cutting;

$\mathrm{h}$ is the thickness of the ring opposite the cut;

$\delta_{c}$ is the change of the ring's diameter after cutting.

The thickness of the thinnest part, where the value of the residual stresses was determined, was accepted as the ring thickness. The diameter of the ring was also determined by the thinnest part.

The upper sign in the formula (1) is used during the removal of the external layers, the bottom sign is used while removing internal layers.

On the surface of the ring

$$
\sigma_{\theta}= \pm \frac{E \delta_{c} h}{\left(1-\mu^{2}\right) D_{a v}^{2}} .
$$

In the latter formula the sign «+» corresponds to the external surface of the ring, while «-» corresponds to the internal one.

We researched the influence of gas-impulsive treatment [1-11] without preliminary heating on the residual stresses in thin-walled ring piped structural elements of aircraft engines, aircraft and airdrome equipment.

The research was done on the equipment which is a gas-jet generator of Galton's whistle type with a cylindrical axisymmetric resonator on the bottom of which a gas pipe is fixed in alignment with the resonator in order to supply compressed air. The opposite end of the gas pipe and a flat round cap make up a slot ring nozzle, the width of which is regulated by changeable annular collars. The captive nut fixes the position of the changeable cutting ring, having a wedge-like profile with the edge directed to the slot nozzle side. Such a generator structure allowed varying the removal of the wedge-like cutting from the nozzle cut, the nozzle slot width, and the reciprocal position of the cutting relative to nozzle edges. The radial jet, being accumulated on the cutting ring, creates the conditions for stimulation of non-stationary resonator filling and emptying, which in its turn causes an oscillatory movement of the radial jet. The resonator was located vertically, and with the help of the threaded joint a cylindrical still-water chamber was fit on its top. It was done to provide an even gas flow velocity distribution on the whole cross-section. The products, which had to undergo a pulsating gas flow treatment, were placed above the stillwater chamber.

The duration of the treatment was from 10 to $20 \mathrm{~min}$ with the oscillation frequency of flow parameters $1-2 \mathrm{kHz}$.

The research of the residual stresses was done by Davidenkov's method applied to the ring samples.

The results of gas-impulsive treatment of the products with the surface stress state, which are made of structural metal materials, are presented in the histogram (Fig. 1). 
The tensile residual stresses are marked by the sign «+», while the compressive stresses are marked by the sign «-»».

The given results demonstrate that the gas-impulsive treatment influences not only the value, but also the sign of the residual stresses on the products' surface, at the same time the value of the stresses decreases.

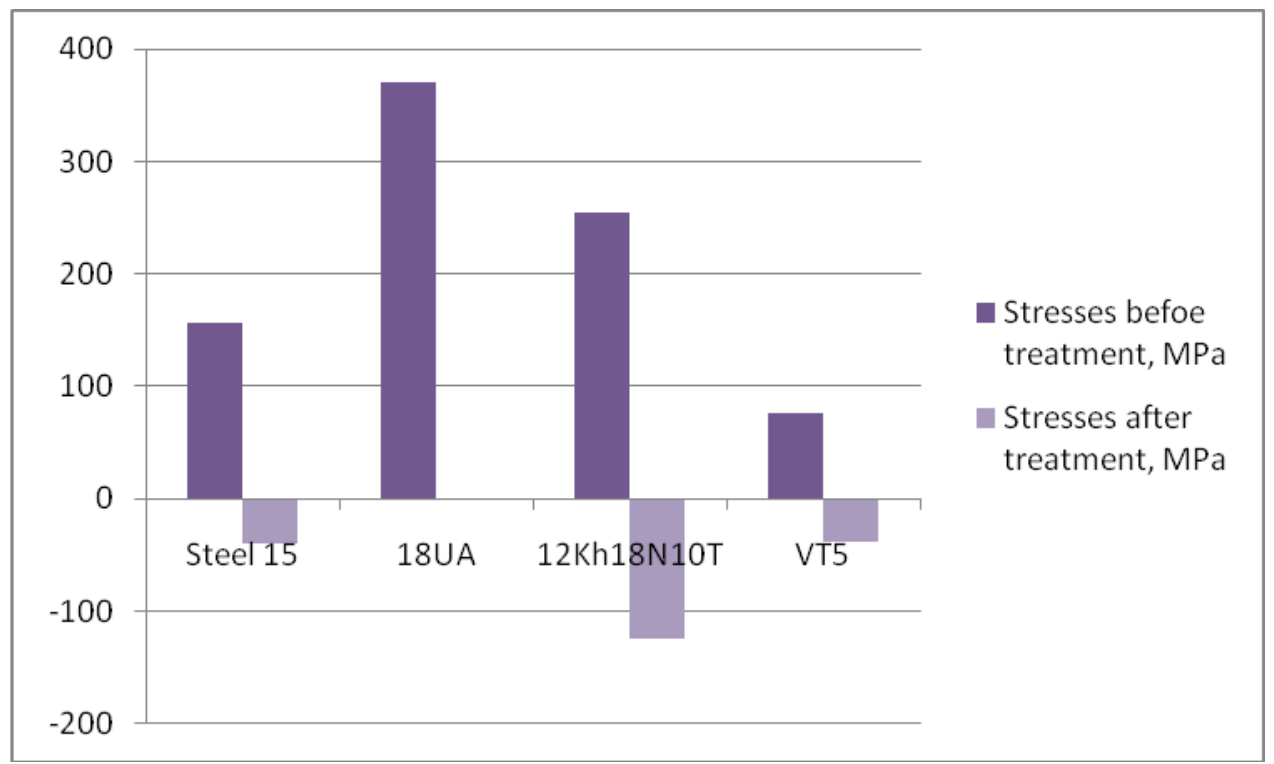

Fig. 1. The level of the tangential residual stresses on the surface of metal ring products before and after the gas-impulsive treatment.

In addition we carried out a more detailed research of the gas-impulsive treatment duration influence on the value of the tangential residual stresses in the surface layers of metal products.

The object of the research was a jointless cold-rolled pipe with the outer diameter of 38 $\mathrm{mm}$ and the wall thickness of $1.1 \mathrm{~mm}$, produced from steel $12 \mathrm{Kh} 18 \mathrm{~N} 9 \mathrm{~T}$, which is used in propulsion engineering.

The width of the cut rings is $10 \mathrm{~mm}$. The tangential tensile stresses on the ring surface were $+183 \mathrm{MPa}$.

The blow-off during 5 minutes by the gas-pulsating flow at ripple frequency of $1130 \mathrm{~Hz}$ and sound pressure of $122 \mathrm{~dB}$ provided the decrease of surface tangential residual stresses by $70 \%$ (to $+55 \mathrm{MPa}$ ), and during 10 minutes by $75 \%$ (to $+46 \mathrm{MPa}$ ) which is a good result if we take into consideration the combination of high toughness of material with the ultimate strength of 1100-1300 MPa (in cold-worked state).

Experimentally we got the following dependence of the tangential residual stresses on the blow-off duration: $2.5 \mathrm{~min}-+293 \mathrm{MPa} ; 5 \min -+55 \mathrm{MPa} ; 7.5 \min -+110 \mathrm{MPa} ; 10$ $\min -+46 \mathrm{MPa}, 12.5 \mathrm{~min}-+82 \mathrm{MPa} ; 15 \mathrm{~min}-+73 \mathrm{Mpa} ; 17.5 \mathrm{~min}-+55 \mathrm{Mpa}$ (Table 1).

The periodic increase of the tangential residual stresses level values may be connected with the decrease of axial and radial residual stresses, having the opposite sign, influence as a result of their relaxation.

Thus, it was found out that there is an optimum duration of the gas-impulsive treatment from the point of view of the level and the sign of the residual stresses on the product surface, and depending on the material, geometric parameters of the sample, and amplitudefrequency of the flow itself. 
Table 1. The values of the tangential residual stresses in the surface layers of the ring samples made from steel $12 \mathrm{Kh} 8 \mathrm{~N} 9 \mathrm{~T}$ depending on the gas-impulsive treatment duration.

\begin{tabular}{|c|c|c|c|c|c|c|c|}
\hline $\begin{array}{c}\text { Treatment duration, } \\
\text { min. }\end{array}$ & 2.5 & 5 & 7.5 & 10 & 12.5 & 15 & 17.5 \\
\hline Residual stresses, MPa & 293 & 55 & 110 & 46 & 82 & 73 & 55 \\
\hline
\end{tabular}

The further continuation of the blow-off makes the process less manufacturable and may lead to the decrease of gas-impulsive treatment positive effect.

As numerous experimental researches showed, the gas-impulsive treatment of metal products, including aircraft structural elements and airdrome equipment, is an effective means of controlling the value and the sign of the residual stresses, having appeared in the process of manufacturing the product, its exploitation and corrective maintenance.

The main factor, determining the value and the sign of the residual stresses, is the blowoff duration.

Taking into consideration that at the initial moment of the blow-off the residual stresses in the surface layers of the product are positive (tensile), i. e. being the most dangerous during exploitation, we may get their zero value during the treatment duration $\mathrm{T} / 4$, where $\mathrm{T}$ is the period of about 20 minutes.

Continuing the gas-impulsive treatment we may get compressive residual stresses on the product surface, which are desirable in many cases, as they increase the steadiness to corrosion and fatigue breakdown. The negative (compressive) residual stresses grow in modulo till the moment, corresponding to $\mathrm{T} / 2$, the modulus of their values is not more than $40 \%$ from the initial value of the residual stresses $\sigma_{\text {reso }}$.

During further treatment of the product the residual stresses, without changing the sign, are decreasing in modulo till zero value (time moment $3 / 4 \mathrm{~T}$ ) and changing the sign into the positive one, reach the maximum value at the time moment $T$, the value of the residual stresses at $\mathrm{T}$ moment is significantly less than the initial one.

The consequence of the blow-off continuation will be the reiteration of the above described process with significantly less values of the residual stresses in comparison to the first period; to the time moment $3 / 4 \mathrm{~T}$ the level of the residual stresses does not practically change and remain being close to zero.

Thus, the process of residual stresses change under gas-impulsive treatment is similar to the process of oscillation damping and can be described by the corresponding equation:

$$
y=A \cdot e^{-a x} \sin \left(\omega x+\varphi_{0}\right)
$$

where $A$ is the initial oscillation amplitude (positive value), $a$ is the damping coefficient, $A \cdot e^{-a x}$ is the instantaneous amplitude, $\left(\omega x+\varphi_{0}\right)$ is the oscillation phase, $\omega=2 \pi / T$ is the cyclic frequency, $\varphi_{0}$ is the initial oscillation phase.

The experimental date processing allowed to get the following empirical formula:

$$
\bar{\sigma}_{\text {residual }}=\sigma_{\text {residual }} / \sigma_{\text {residual } 0}=e^{-0,1 \tau} \cdot \cos (\omega \tau)
$$

Where $\tau$ is the time of the blow-off.

The dependence $\sigma_{\text {ras }} / \sigma_{\text {ress }}$ on the blow-off duration is presented in Fig. 2 . 


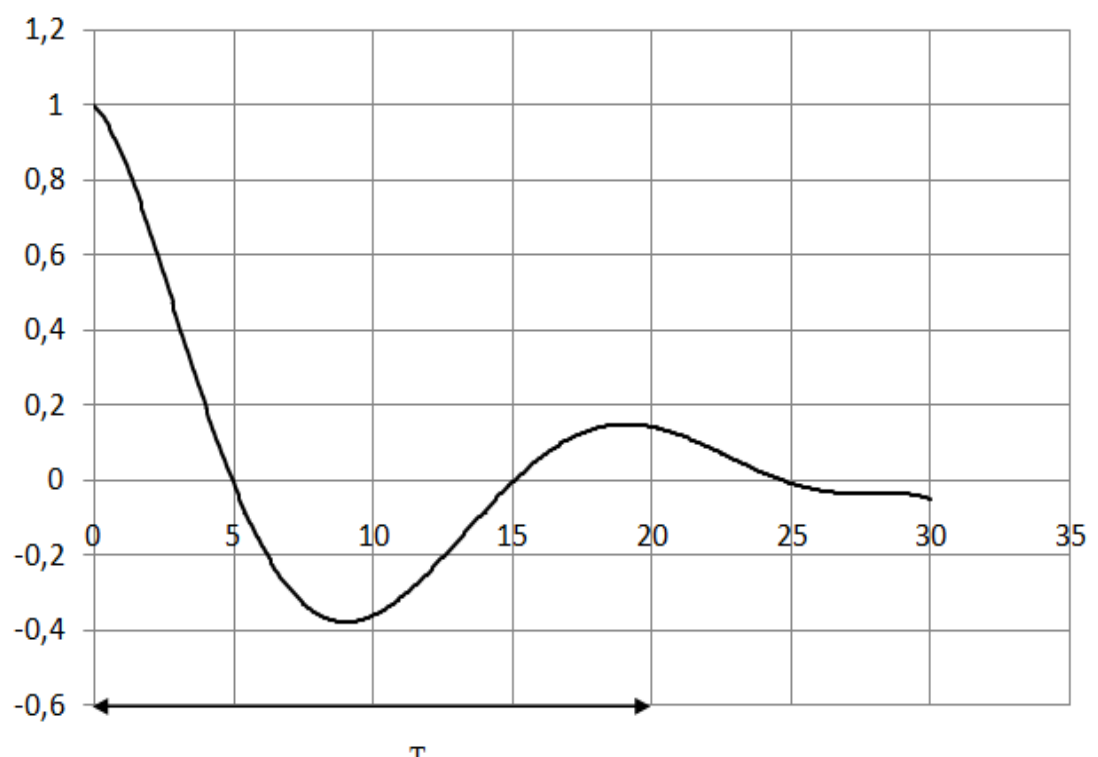

$\mathrm{T}$

Fig. 2. The dependence of the residual stresses values relation to their initial value on the duration of pulsating subsonic gas flow treatment.

The rings of the rolling bearings underwent the gas-impulsive treatment; despite the displacement of structural steels in the aircraft structures by titanic alloys and other materials, the bearings remain steel. The rings of the rolling bearings during exploitation undergo cyclic alternate loads; that is why to provide a prolonged bearing exploitation it is necessary to exclude undesirable tensile stresses on its rings surface. This can be achieved by the gas-impulsive treatment. The bearings' rings undergo hardening by martensite with the further low tempering for preservation of high solidity and durability. The strained residual stresses often appear on the surface of the bearings' rings which lower their durability. Similar stresses may appear as a result of rings' surface polishing process in order to give the necessary smoothness to their surface.

The tangential tensile stresses on the surface of the raw ring were $+27 \mathrm{MPa}$; and as a result of the blow-off during 5 minutes they showed the value $+13.5 \mathrm{MPa}$; after the gasimpulsive treatment during 10 minutes the residual stresses on the surface became compressive with the value of $-27 \mathrm{MPa}$; and after 15 minutes of treatment the stresses had a zero value (see the graph in Fig. 3).

This manifested the dependence character of residual stresses level on the product surface on the gas-impulsive treatment duration, which is already known from the previous researches. 


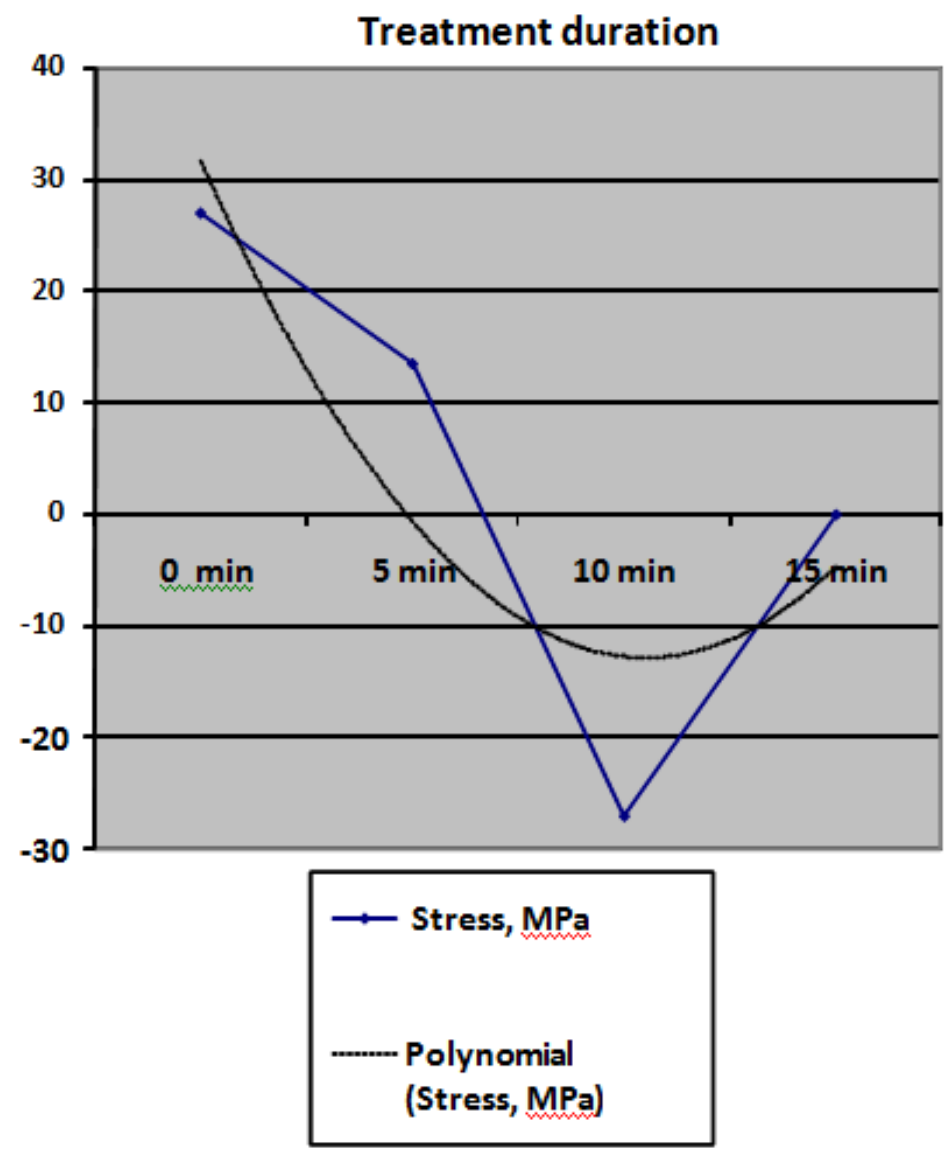

Fig. 3. The level of tangential residual stresses on the surface of the rolling bearing rings made from steel ShKh15 depending on the gad-impulsive treatment duration.

Further the ring samples of aluminum alloy AMg6 (the outer diameter is $33 \mathrm{~mm}$, the ring width is $7 \mathrm{~mm}$, the wall thickness is $1.2 \mathrm{~mm}$ ) underwent subsonic pulsating air flow treatment. The tangential residual tensile stresses were $122 \mathrm{MPa}$ on the surface and were nullified after pulsating gas flow treatment.

The results of the research have shown that the use of pulsating subsonic air flows has prospects in order to control residual stresses in aircraft metal structural elements.

\section{References}

1. Y. A. Kuznetsov, A. V. Kolomeichenko, V. V. Goncharenko, I. N. Kravchenko, Mater. Sci. Forum. 968, 153-160 (2019)

2. A. V. Kolomeichenko, S. N. Sharifullin, Yu. A. Kuznetsov, V. N. Logachev, N. V. Titov, J. Phys. Conf. Ser. 1058, 012074 (2018)

3. Q. P. Tran, Y. C. Kuo, J. K. Sun, J. L. He, T. S. Chin, Surf. Coat. Technol. 303, 61-67 (2016)

4. A. A. Filippov, G. V. Pachurin, V. I. Naumov, N. A. Kuzmin, Metallurgist 59, 810-815 (2016)

5. Y. N. Smyrnov, V. A. Skliar, V. A. Belevitin, R. A. Shmyglya, O. Y. Smyrnov, Steel Transl. 46, 325-328 (2016) 
6. A. N. Smirnov, S. V. Kuberskii, E. N. Smirnov, A. P. Verzilov, E. N. Maksaev, Steel Transl. 47 (7), 476-482 (2017)

7. E. N. Smirnov, V. A. Sklyar, A. N. Smirnov, V. A. Belevitin, S. P. Erońko, R. E. Pivovarov, Steel Transl. 48 (6), 381-387 (2018)

8. G. V. Kovalenko, D. A. Ivanov, Advances in Engineering Research: Proceedings of the International Conference on Aviamechanical Engineering and Transport (AviaENT 2019) 188, 179-183 (Atlantis Press, 2019)

9. D.A. Ivanov, O.N. Zasukhin, Russian Metallurgy (Metally) 13, 1522-1525 (2019)

10. D.A. Ivanov, O.N. Zasukhin, Method of hardening heat treatment of carbon tool steels, Patent 2557841 C2 Russian Federation, Bul. 21, (51) IPC C21D 1/04 (declared. 13.09.20132, publ. 27.07.2015)

11. D.A. Ivanov, O.N. Zasukhin, Method of increasing the resistance of metal-cutting tools made of high-speed steel (Patent 2580767 C2 Russian Federation, Bul. 10 (51) IPC C21D 9/22, decl. 24.12.2013, publ. 10.04.2016) 